\title{
Long-term change in incidence and risk factors of cirrhosis and hepatocellular carcinoma in Crete, Greece: a 25-year study
}

\author{
Spyridon A. Karageorgos ${ }^{a}$, Soultana Stratakou ${ }^{a}$, Mairi Koulentaki ${ }^{a}$, Argyro Voumvouraki ${ }^{a}$, \\ Aikaterini Mantaka ${ }^{a}$, Dimitrios Samonakis ${ }^{a}$, George Notas ${ }^{b}$, Elias A. Kouroumalis ${ }^{a}$
}

\begin{abstract}
Background No sequential long-term data exist for Greece on the etiological evolution and incidence of cirrhosis and hepatocellular carcinoma. Therefore, we studied their etiological evolution over a period of 25 years in the island of Crete.

Methods We studied 812 cases of cirrhosis (561 male, median age 69 years) and 321 cases of hepatocellular carcinoma (234 male, median age 70 years) from the database of our Center. Cases were classified into five-year periods according to incidence and etiology (hepatitis B, hepatitis C, alcohol, alcohol plus viral, and non-alcoholic fatty liver disease).

Results Overall, there was an increase in the incidence of hepatocellular carcinoma. A significant fourfold reduction in the incidence of hepatitis C-related cirrhosis was observed, which was degraded from first to third place as a risk factor for cirrhosis. Alcohol gradually became the first risk factor in cirrhosis (1990-94: 36.1\%, 2010-14: 52.3\%) and carcinoma, while the steepest increase in incidence of cirrhosis and carcinoma was associated with non-alcoholic fatty liver disease.

Conclusions The incidence of cirrhosis remained constant over the years, but the incidence of hepatocellular carcinoma increased during the last decade. Risk factors for cirrhosis and hepatocellular carcinoma have changed over the past 25 years in Crete. The initial high hepatitis $\mathrm{C}$ virus association has significantly decreased, with alcohol now ranking first among risk factors. Non-alcoholic fatty liver disease is continually increasing and is a prominent risk factor for cirrhosis and hepatocellular carcinoma.
\end{abstract}

Keywords Hepatocellular carcinoma, cirrhosis, non-alcoholic fatty liver disease, HCV, hepatocellular carcinoma

Ann Gastroenterol 2017; 30 (3): 1-7

\section{Introduction}

Liver cirrhosis and hepatocellular carcinoma (HCC) constitute major causes of morbidity and mortality

\footnotetext{
${ }^{a}$ Department of Gastroenterology and Hepatology, University Hospital of Heraklion and University of Crete School of Medicine (Spyridon A. Karageorgos, Soultana Stratakou, Mairi Koulentaki, Argyro Voumvouraki, Aikaterini Mantaka, Dimitrios Samonakis, Elias A. Kouroumalis); 'baboratory of Experimental Endocrinology, University of Crete School of Medicine (George Notas), Heraklion, Crete, Greece
}

\section{Conflict of Interest: None}

Correspondence to: Associate Prof. George Notas, MD, PhD, Laboratory of Experimental Endocrinology, University of Crete School of Medicine, Heraklion, PO Box 2208, 71003, Crete, Greece, Tel: +30 2810 394556, Fax: +30 2810 394556, e-mail: gnotas@uoc.gr

Received 12 September 2016; accepted 10 January 2017; published online 23 March 2017

DOI: https://doi.org/10.20524/aog.2017.0135 worldwide [1-3]. Cirrhosis is the end result of virtually all chronic liver diseases. An asymptomatic phase, referred to as compensated cirrhosis, is followed by the development of complications of portal hypertension, namely ascites, variceal bleeding and hepatic encephalopathy, collectively termed decompensated cirrhosis. The transition occurs at a rate of $5-7 \%$ per year [4]. Liver cancer is the sixth most common malignancy, the third cause of cancer-related death, and accounts for $7 \%$ of all cancers. HCC represents the majority of primary liver cancers and has a high mortality rate [1,2]. Many causes have been identified for the development of cirrhosis and HCC. Chronic hepatitis B, hepatitis C infection and high alcohol consumption remain the most frequent causes [5]. Over the last years, non-alcoholic fatty liver disease (NAFLD) has emerged as a major etiological factor for the development of cirrhosis and HCC worldwide [5-7].

Only a limited number of studies have reported changes in the etiology of HCC; until now, there have been no sequential long-term data regarding the changes in the etiology of cirrhosis and HCC in Greece. The aim of this study was to evaluate the etiological evolution of cirrhosis and HCC over a 
period of 25 years in the genetically homogeneous population of the island of Crete, which has 623,065 inhabitants (2011 census) and low immigration rates. To determine whether a change in HCV-associated cirrhosis was accompanied by changes in $\mathrm{HCV}$ genotypes, we also examined genotypes in two different time periods.

\section{Patients and methods}

The study conformed to the principles of the Declaration of Helsinki and was approved by the Ethics Committee of the University Hospital of Heraklion. The study was performed in a tertiary university hospital, which is the reference center for liver disease on the island of Crete, in southern Greece. Patients included in the study were either diagnosed with cirrhosis/ HCC on the basis of their symptoms, or referred to our unit because of laboratory findings related to significant liver disease (significantly elevated liver function tests, diagnosis of hepatitis B [HBV] or hepatitis C [HCV] seropositivity, etc.). The population of 623,065 inhabitants shares a relatively homogenous background and there is still a low migration rate. Recorded data included age, sex, date of diagnosis of HCC, date of diagnosis of cirrhosis, etiology of HCC, etiology of cirrhosis, presenting symptoms and histological pattern at diagnosis. All patients were recorded only once at the time of their initial diagnosis.

Diagnosis was confirmed by liver biopsy in $57 \%$ of cirrhotic patients (mostly compensated cirrhosis), while clinical evidence of decompensation (present in $62 \%$ of cases when entered into the database), combined with endoscopic and radiological findings, was used for diagnosis in the remaining cases. Diagnosis of NAFLD-associated cirrhosis was based on the absence of alcohol consumption or viral markers and the presence of either overt diabetes or the presence of features of the metabolic syndrome. In $73 \%$ of NAFLD cases, liver biopsy was performed to verify the diagnosis.

The diagnosis of HCC was established by liver biopsy with a histological pattern of HCC ( $62 \%$ of patients), or by 2 dynamic radiological methods (computed tomography, magnetic resonance imaging, SonoVue-enhanced ultrasound). All cases were then classified into five-year periods according to etiology (HBV, HCV, alcohol-related, alcohol plus viral infection, and NAFLD). Most of the HCC patients were derived from our cirrhotic cohort. Patients with a first diagnosis of cirrhosis and HCC at their initial visit were included in both lists. Thirty patients did not have evidence of cirrhosis based on liver biopsy, radiological and clinical data. Therefore, the total number of patients was 842: 812 cirrhotics (291 of them diagnosed at some point with HCC); and 30 patients with HCC but no evidence of cirrhosis.

No etiology could be identified in 66 cases of cirrhosis (cryptogenic), which comprised $8 \%$ of all cirrhosis cases in the 25 years of the study. Patients with primary biliary cirrhosis and autoimmune hepatitis were excluded from this study, as they have a discrete clinical course and in the case of primary biliary cirrhosis our experience has been reported elsewhere [8]. Therefore, the final analysis included 321 HCC patients (291 of whom with cirrhosis) and 746 patients with cirrhosis without HCC.

$\mathrm{HCV}$ genotypes were assessed in 302 anti-HCV positive, HCV RNA positive patients with histological confirmation of chronic hepatitis, tested between 2005 and 2009, and were compared with data from a group of 102 patients from the time period 1995-99. Genotyping was performed using the Abbott Real-Time HCV Genotyping kit (Abbott Hellas, Athens, Greece).

All comparisons were performed using the chi-square test. Incidence was expressed as number of new cases per five years per 100,000 population. Population data over the past 25 years were collected from the Greek Statistical Authority.

\section{Results}

A total of 812 cases of cirrhosis (561 male, representing $69.1 \%$ of the study population, median age 69 years, range 33-86) and 321 cases of HCC (234 male, representing 73.0\% of the study population, median age 70 years, range 46-89) were recorded. Two hundred ninety-one HCC patients also had cirrhosis, while 30 HCC patients were diagnosed with HCC without evidence of cirrhosis $(291+30=321$ patients with HCC in total). Therefore, these $291 \mathrm{HCC}$ cases are also present in the cirrhosis patient list, even if HCC and cirrhosis were diagnosed at the initial visit. Among the 812 cirrhotic patients, there were 66 in whom a diagnosis could not be achieved due to several reasons (e.g. lost to follow up, death, non-diagnostic results, etc.) and these were not included in further analysis. Demographic and other patient characteristics are presented in Table 1. Data were classified into 5 -year periods according to etiology. There were 746 cases of cirrhosis related to HBV, $\mathrm{HCV}$, alcohol, alcohol plus viral infection and NAFLD, while the remaining cases had insufficient data to reach a diagnosis and could not be used for further analysis.

In patients with cirrhosis, there was a relative uniformity in the incidence over the years with a peak during 1995-99 due to increased detection of cases related to viral cirrhosis (Table 2 and Fig. 1A). The incidence of HBV-related cirrhosis remained relatively constant, apart from the period 1995-99 (Fig. 1B, 2A). There was a significant decrease in the incidence of HCV-related cirrhosis, which was four times lower in the last five years of the study compared with the initial period. The incidence of alcoholic cirrhosis and NAFLD-related cirrhosis increased during our study, with the incidence of NAFLD-associated cirrhosis in the last period of the study being six times greater than the initial value (Table 2, Fig. 1B). When etiology was assessed as a percentage of total cases, significant changes were observed over the 25-year period (Table 2, Fig. 2B). HBV-related cases were relatively constant (1990-1994: 8.3\%, 1995-1999: 20.4\%, 2000-2004: 13.9\%, 20052009: 12.1\%, 2010-2014: 7.3\%), while HCV-related cirrhosis decreased from $44.4 \%$ in $1990-1994$ to $13.0 \%$ in $2010-2014$ $(\mathrm{P}<0.001,95 \% \mathrm{CI} 8-22 \%)$. On the other hand alcohol-related cirrhosis increased over the years (1990-1994: 36.1\%, 2010- 
2014: $52.3 \% \mathrm{P}<0.001$, 95\%CI 11-35\%) with a concomitant increase in cases of NAFLD-related cirrhosis (1990-1994: 1.4\%, 2010-2014: 15.5\%; $\mathrm{P}<0.001$, 95\%CI 9-21\%).

Both the incidence and the etiology of HCC changed over the last 25 years in the island of Crete. Incidence rose from 6.0 new cases per 100,000 of population in the first five-year period to 16.8 in the last five years (Table 3, Fig. 1A). The change was mostly attributable to a gradual increase in the incidence of HBV- and alcohol-related cases, especially during the last decade. There was also an impressive increase in the incidence of NAFLD-related cases (Fig. 2C). A non-significant increase in the incidence of HCV-related HCC was also observed (Table 3, Fig. 1C).

When etiologies were expressed as percentages of the total number of cases, there was no significant change in the percentages of HBV- and alcohol-related HCC over all fiveyear periods. Although the absolute number of HCV-related HCC cases remained relatively constant, HCV ranked third as a risk factor in the last study period, behind HBV and alcohol. This was in sharp contrast with the early 1990s, when almost half the HCC cases were HCV-associated (Table 3, Fig. 2D).

Table 1 Patients' characteristics. Values are expressed as mean \pm standard deviation, unless otherwise stated

\begin{tabular}{lcc}
\hline Patients' characteristics & Cirrhosis $(\mathrm{n}=812)$ & HCC $(\mathrm{n}=321)$ \\
\hline Age (median, range) & $69(33-86)$ & $70(46-86)$ \\
Male sex & 561 & 234 \\
ALT (IU/L) & $53 \pm 17$ & $48 \pm 23$ \\
Bilirubin (mg/dL) & $2.5 \pm 1.8$ & $1.8 \pm 1.3$ \\
$\gamma$ GT (<35 IU) & $38 \pm 22$ & $77 \pm 37$ \\
ALP (<120 IU) & $108 \pm 36$ & $182 \pm 53$ \\
aFP>400 ng/dL (\%) & & 53 \\
Child-Turcotte-Pugh & & \\
score (\%) & & \\
A & 23 & 28 \\
B & 32 & 52 \\
C & 45 & 20 \\
\hline
\end{tabular}

$\alpha F P, \alpha$-fetoprotein; ALP, alkaline phosphatase; $\gamma G T, \gamma$-glutamyltransferase; HCC, hepatocellular carcinoma; ALT, alanine transaminase
Thus, although in the period 1990-1994 HCV-related HCC represented $50.0 \%$ of all cases, in 1995-1999 it was $42.9 \%$, in 2000-2004 it was $24.4 \%(\mathrm{P}<0.05)$, in $2005-2009$ it was $24.7 \%(\mathrm{P}<0.001)$ and in $2010-2014$ it was $21.2 \%(\mathrm{P}<0.001$, 95\%CI 11-47\%). Interestingly, we also identified a change in HCV genotypes between the early years (1994-2000) and the late years (2007-2009) of our study, as presented in Table 4. Genotype 1 decreased significantly over the years while genotype 3 increased considerably.

A very interesting finding of our study was the significant increase in NAFLD-related HCC over the years. From 1990 to 2004 NAFLD-related HCC was almost nonexistent, while in 2005-2010 16.5\% of HCC cases were attributed to this disease and during 2010-2014 this reached $23.1 \%$ of all cases (95\% CI 13-31\%, Table 3, Fig. 2D). Thus, NAFLD-related HCC displayed the steepest increase of all the diseases studied (Fig. 1C).

Of all HCC cases, 9.3\% had no cirrhotic histology. In these cases histology showed that, in addition to the malignant tissue, there was a non-malignant part without evidence of cirrhosis, while in patients where the diagnosis was reached through radiology, these patients had no clinical evidence suggestive of cirrhosis or portal hypertension (no varices on endoscopy, no splenomegaly, normal blood counts). Furthermore, 54\% of them were NAFLD-associated HCC and the remaining were alcohol-related (34\%), or they had known chronic viral hepatitis (12\%).

\section{Discussion}

Liver cirrhosis and HCC are major causes of morbidity and mortality worldwide [1-3] and the risk factors that have been linked to their development have been extensively studied. Cirrhosis has been mainly linked to chronic HBV or HCV infection, and to alcohol consumption [9], metabolic liver diseases including NAFLD, hereditary hemochromatosis, Wilson's disease, a1-antitrypsin deficiency, primary biliary cirrhosis and primary sclerosing cholangitis [10]. Major risk factors for the development of HCC include chronic infection with HBV and HCV (80-90\% of HCC cases have been reported to be associated with underlying HBV or HCV cirrhosis [11,12]), alcoholic liver disease, environmental food

Table 2 Changes in the incidence, the number and the etiological distribution of new cases of cirrhosis between 1990 and 2014

\begin{tabular}{|c|c|c|c|c|c|}
\hline \multirow[t]{2}{*}{ Etiology } & \multicolumn{5}{|c|}{ Incidence of cirrhosis, n (\%) } \\
\hline & 1990-1994 & 1995-1999 & $2000-2004$ & 2005-2009 & $2010-2014$ \\
\hline $\mathrm{HBV}$ & $2(12-8.3)$ & $7.5(46-20.4)$ & $2.8(17-13.9)$ & $2.6(16-12.1)$ & $1.5(9-7.3)$ \\
\hline $\mathrm{HCV}$ & $10.6(64-44.5)$ & $15.8(95-42.2)$ & $5.4(33-27.1)$ & $3.3(20-15.1)$ & $2.6(16-13)$ \\
\hline ALC & $8.6(52-36.1)$ & $9.7(58-25.6)$ & $7.9(48-39.3)$ & $10.8(66-50)$ & $11(68-52.3)$ \\
\hline $\mathrm{ALC}+\mathrm{VIR}$ & $2.3(14-9.8)$ & $3.7(22-9.8)$ & $2.2(13-10.6)$ & $2.3(14-10.6)$ & $1.8(11-8.9)$ \\
\hline NAFLD & $0.4(2-1.4)$ & $0.7(4-1.8)$ & $1.8(11-13.1)$ & $2.6(16-14.4)$ & $3.1(19-15.5)$ \\
\hline Total & $24(144-100)$ & $37.5(225-100)$ & $20(122-100)$ & $21.6(132-100)$ & $19.9(123-100)$ \\
\hline
\end{tabular}

HBV, hepatitis B; HCV, hepatitis C; ALC, alcohol; ALC+VIR, alcohol and viral hepatitis; NAFLD, non-alcoholic fatty liver disease 


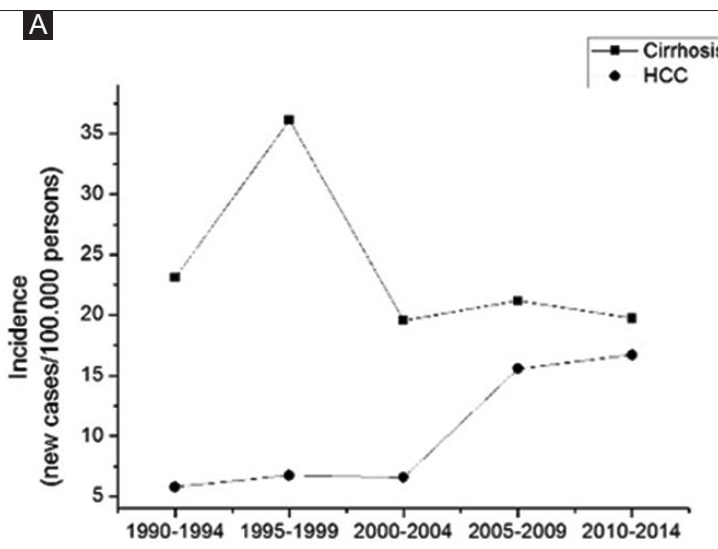

B

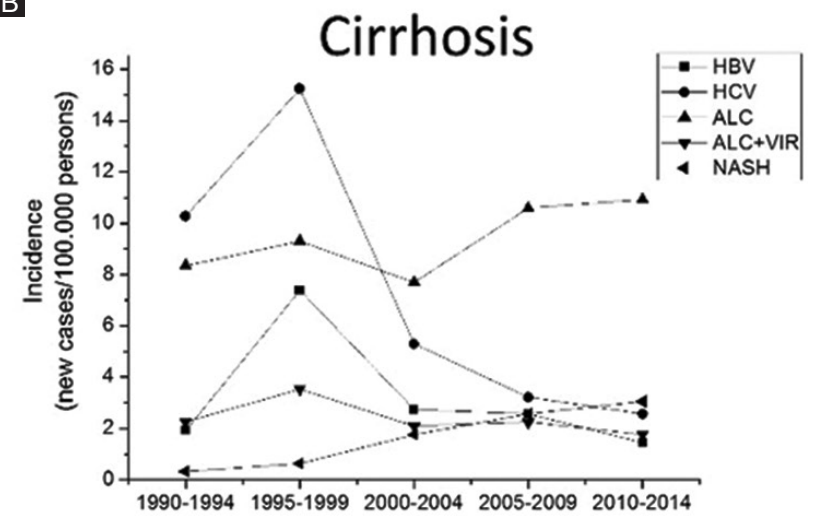

C

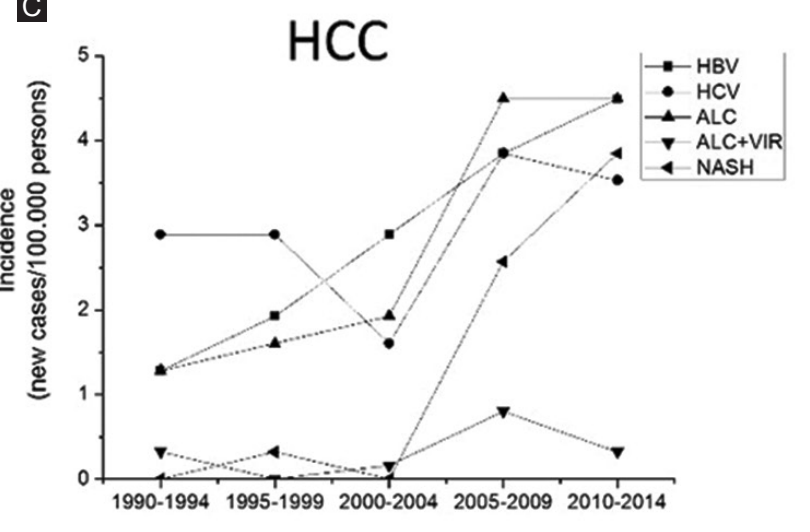

Figure 1 (A) Overall incidence of cirrhosis and hepatocellular carcinoma (HCC) over 25 years. (B) Etiology-based comparison of the changes in the incidence of new cases of cirrhosis. (C) Etiology-based comparison of the changes in the incidence of new cases of HCC over the 25 years of the study

Table 3 Changes in the incidence, the number and the etiological distribution of hepatocellular carcinoma (HCC) between 1990 and 2014 Etiology Incidence of HCC, $\mathrm{n}(\%)$

\begin{tabular}{lccccc}
\cline { 2 - 5 } & $1990-1994$ & $1995-1999$ & $2000-2004$ & $2005-2009$ & $2010-2014$ \\
\hline HBV & $1.3(8-22.2)$ & $2(12-28.6)$ & $2.9(18-43.9)$ & $3.9(24-24.7)$ & $4.5(28-26.9)$ \\
HCV & $3(18-50.0)$ & $3(18-42.9)$ & $1.7(10-24.4)$ & $3.9(24-24.7)$ & $3.6(22-21.2)$ \\
ALC & $1.3(8-22.2)$ & $1.7(10-23.8)$ & $2(12-29.3)$ & $4.6(28-28.9)$ & $4.5(28-26.9)$ \\
ALC+VIR & $0.3(2-5.6)$ & $0(0-0.0)$ & $0.2(1-2.4)$ & $0.8(5-5.2)$ & $0.3(2-1.9)$ \\
NAFLD & $0(0-0.0)$ & $0.4(2-4.8)$ & $0(0-0.0)$ & $2.7(16-16.5)$ & $3.7(24-23.1)$ \\
Total & $6(36-100.0)$ & $7(42-100.0)$ & $6.7(41-100.0)$ & $15.9(97-100.0)$ & $16.8(104-100.0)$ \\
\hline
\end{tabular}

ALC, alcohol; $A L C+V I R$, alcohol and viral hepatitis; $H B V$, hepatitis $B ; H C V$, hepatitis $C ; N A F L D$, non-alcoholic fatty liver disease

contaminants (aflatoxins) and recently NAFLD [5,6]. Diabetes, obesity, tobacco, and oral contraceptives have also been considered as risk factors for HCC [13-16]. Our data show that, despite the existence of a relatively large number of HCC cases unrelated to cirrhosis, it is evident that the presence of cirrhosis remains the main risk factor for the development of HCC.

In our study, the overall incidence of cirrhosis has remained relatively constant over the years, but the incidence of HCC has almost tripled over the 25 -year period. A recent paper from neighboring Albania reported that the incidence of HCC almost doubled within the last 15 years but, in contrast to our data, almost half of their cases were HBV-related [17]. It should be noted that 15 years ago, the incidence of HCC was similar in Albania and Crete at approximately 6.5 new cases per 100,000 [17]. It is unlikely that the difference in HCC incidence is due to better diagnostic tools during the last periods of observation, since most patients with HCC (62\%) were diagnosed by liver biopsy at the beginning of the study. 

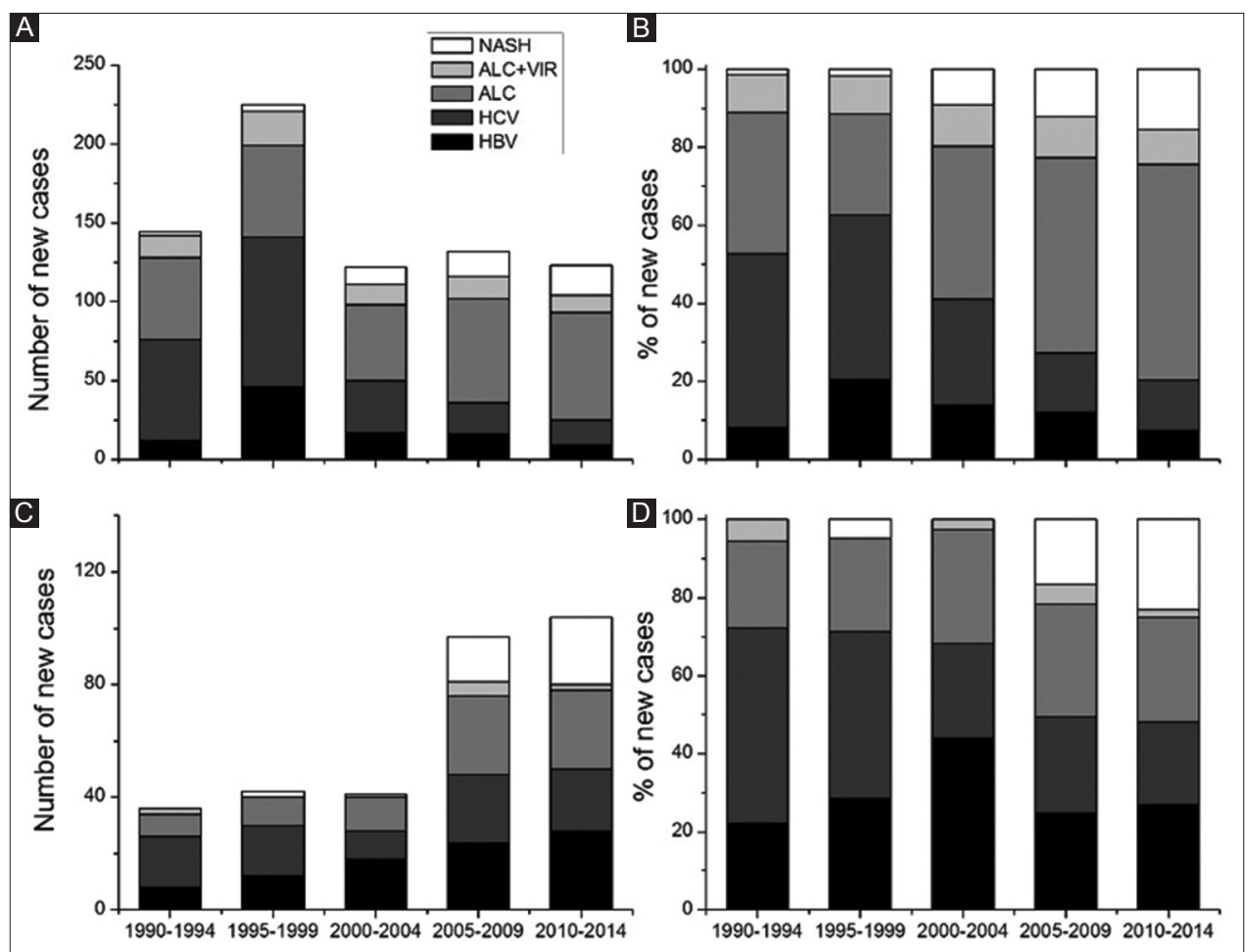

Figure 2 (A) Stacked bars of the number of new cirrhosis cases over the 25 years of the study according to etiology. (B) Stack bars of etiological representation (\% of the total) in cirrhosis. (C) Stacked bars of the number of new hepatocellular carcinoma (HCC) cases over the 25 years of the study according to etiology. (D) Stack bars of etiological representation (\% of the total) in HCC

$A L C$, alcohol; $A L C+V I R$, alcohol and viral hepatitis; HBV, hepatitis B virus; HCV, hepatitis C virus; NASH, non-alcoholic steatohepatitis

Table 4 The shift in hepatitis $\mathrm{C}$ virus genotypes over the years

\begin{tabular}{lccc}
\hline Variable & $1994-1997$ & $2007-2010$ & $\mathrm{P}$ \\
\hline Patients (M/F) & $102(49 / 53)$ & $226(154 / 72)$ & \\
Age (mean \pm SD) & $51.5 \pm 15.8$ & $46.3 \pm 16.2$ & \\
Age median & $56(16-77)$ & $43(18-89)$ & \\
Genotype & & & \\
1 & $64 \%$ & $38 \%$ & $<0.01$ \\
2 & $13 \%$ & $6.6 \%$ & \\
3 & $18 \%$ & $45.5 \%$ & $<0.01$ \\
4 & $5 \%$ & $8.4 \%$ & \\
\hline
\end{tabular}

M, male; F, female; SD, standard deviation

Likewise, dynamic radiology was used from the beginning of the study (contrast-enhanced ultrasound was used as early as 1997 by the first radiologist to use the method in Greece).

All patients with HBV-related cirrhosis were treated with oral antiviral therapy at presentation, while no patient with HCVrelated cirrhosis was offered antiviral therapy with an interferonbased scheme. Although antiviral therapy may influence the outcome and the complication rate of patients with virus-related chronic liver disease, there seems to be no effect on the increasing incidence of HBV-associated HCC (Table 2), while the incidence of HCV-associated cirrhosis remained constant.

Chronic HBV infection is found in $5 \%$ of the world's population, while the global prevalence of $\mathrm{HCV}$ is $2 \%$, with a large variation in different geographical areas [11]. In Crete, we have reported HCV infection to be more prevalent than $\mathrm{HBV}$, but while HCV remained relatively constant, HBV prevalence almost tripled in two studies performed in two areas of the island (rural and urban) in two different time periods separated by a 15 -year interval $[18,19]$. Despite this increase, according to the data presented here, HBV-related cirrhosis in Crete remained constant over the years, while the incidence of HBV-related HCC increased. This might reflect that current therapies are more effective in controlling the evolution of the disease to cirrhosis and less effective against the direct carcinogenic effects of HBV. Current therapies do not influence the cccDNA pool in the nucleus and have been found to reduce the risk of HCC, mainly in patients with active disease and those with advanced fibrosis or cirrhosis [20]. More studies are needed in order to clarify whether the risk reduction for HCC in non-cirrhotic, yet treated HBV patients is similar to that of the non-infected population. Furthermore, the role of HBV as a predisposing factor for cirrhosis and HCC will most likely decrease during the coming decades, because of the widespread use of the $\mathrm{HBV}$ vaccine in newborns in Greece [12].

We also report here that HCV-related cirrhosis has decreased over the 25-year period of our study and HCVrelated HCC has decreased as a percentage of the overall HCC burden over the same period, although the total cases increased slightly, but not significantly. One explanation for this would be that the reduction in HCC incidence coincides with the 
reduction in HCV-related cirrhosis. However, this was not the case in a study from Italy, where from 1987 to 2008 there was no major change in the incidence of HBV-related HCC but there was an increase in HCV- and alcohol-related HCC [21]. A study of the population of Athens reported similar findings, with a more than twofold increase in HCV-related HCC [22]. On the other hand, the total burden of HCV infection seems to be decreasing in the United States, since the number of cases of chronic HCV infections decreased from 3.2 million in 2001 to 2.3 million in 2013 [23]. This was attributed to the widespread implementation of screening and treatment. Increased access to proper diagnostic modalities for $\mathrm{HBV}$ and $\mathrm{HCV}$ has shown that the epidemiology of viral hepatitis is continuously changing.

Another possible explanation for the decreasing incidence of HCV-related HCC could be the shift in HCV genotypes from genotype 1 towards genotype 3 over the years. This is usually associated with a younger age and intravenous drug use. Although reports associating HCV genotype and HCC risk are inconsistent, some of them and a meta-analysis reported that patients infected with HCV genotype $1 \mathrm{~b}$ had an almost 2-fold greater risk of developing HCC than patients with other HCV genotypes [24,25]. Contrariwise, genotype 3 has recently also been recognized as a risk factor for HCC [26]. One additional explanation is that the genotype 3-associated HCC mostly appears in younger patients, usually intravenous drug users, and therefore the time elapsed was not long enough for the HCC to become apparent. More epidemiological data from large cohorts, extended over a more protracted time, are needed in order to identify the relationship between the changing incidence of HCV genotypes and HCC risk.

Alcohol consumption is a predominant etiological factor in the pathogenesis of chronic liver diseases worldwide [27]. According to the findings of the present study this was not the case in Crete in the early nineties, but within the study period the incidence of alcohol-related cirrhosis has increased significantly and alcohol is now the main etiology for cirrhosis development. This could reflect the increasing role of alcohol consumption as an etiology for liver diseases reported in other parts of the world [21]. Although no systematic data concerning alcohol consumption exist in Crete, it is our clinical impression that an increase in absolute ethanol consumption is only one of the reasons for this change. Another reason could be that, during the last twenty years, there has been a change in drinking habits: although older generations consumed alcohol only with food, in recent years alcohol is increasingly consumed without food.

NAFLD has emerged as a major etiological factor for the development of cirrhosis and HCC in many parts of the world. It is a heterogeneous disease which consists of a wide spectrum of different histologic patterns characterized by micro- or macrovesicular hepatic steatosis and ballooning of hepatocytes. NAFLD is now recognized as the liver manifestation of the metabolic syndrome and is a major cause of liver-related morbidity and mortality [28]. NAFLD and non-alcoholic steatohepatitis (NASH) were under-recognized during the early 90 s, and this is probably reflected to some extent in our data and in the data from studies before the wide recognition of NAFLD/NASH as a major predisposing factor for cirrhosis and HCC. Today, NASH is believed to account for the majority of cirrhosis cases, previously considered cryptogenic, and it is accepted that HCC can complicate NASH even if there is no cirrhosis [29-31]. Although efforts to screen for NAFLD/NASH in the general population are limited by the inaccuracy of existing noninvasive tools and the limitations of self-reported ethanol consumption history [32], a recent cohort study in United States revealed a stable incidence rate of NAFLD (2.2$3.2 \%$ ) whereas the prevalence increased from $6.3 \%$ in 2003 to $17.6 \%$ in 2011. Both incidence and prevalence are increasing in patients younger than 45 years old in comparison with older patients [33]. Despite all the limitations, some studies suggest that $20-30 \%$ of individuals in Western countries have NAFLD [34]. Our findings show a disturbing increase in NAFLD-related cirrhosis and HCC in the population of Crete within the last 25 years, which is in concordance with the reported data. Given the increasing prevalence of NAFLD in western societies, NAFLD may become the major cause of cirrhosis and HCC in our study population in the next few years. The extremely large increase in NAFLD-associated HCC during the last 10 years of the study is difficult to explain. Although some selection bias cannot be excluded, we believe that this is a real phenomenon, since it parallels a large increase in outpatient visits due to non-cirrhotic NAFLD. It should be noted that $62 \%$ of liver biopsies of NAFLD patients had

\section{Summary Box}

\section{What is already known:}

- Liver cirrhosis and hepatocellular carcinoma (HCC) constitute major causes of morbidity and mortality worldwide

- The commonest causes of cirrhosis and HCC are hepatitis $\mathrm{B}$ and hepatitis $\mathrm{C}$ infections and high alcohol consumption

- Over the last years, non-alcoholic fatty liver disease (NAFLD) has emerged as a major etiological factor for the development of cirrhosis and HCC worldwide

\section{What the new findings are:}

- Although the incidence of cirrhosis has been constant over the years, the incidence of HCC has increased during the last decade

- The risk factors for cirrhosis and HCC have changed over the past 25 years in Crete

- The initial high hepatitis $\mathrm{C}$ virus association has decreased significantly, with alcohol now ranking first among the risk factors

- NAFLD is continually increasing in incidence and is a prominent risk factor for cirrhosis and HCC 
histological signs of NASH. Moreover, it seems that obesity has increased in the Cretan population, although data extended over time do not exist. This obviously requires further studies.

A limitation of our study is that it was a single-center study, whereas a number of cirrhotic and HCC patients on the island of Crete have been diagnosed and followed in other hospitals on the island. Therefore, although our data demonstrate a clear epidemiological trend, they cannot be considered representative of the entire Cretan population. However, since our center is the only reference center on the island, it is likely that most cirrhotic patients on the island were referred to our outpatient department at some point in time.

In conclusion, our study revealed that the risk factors for cirrhosis and HCC have changed considerably over the last 25 years in Crete. The initial high association of HCV with cirrhosis has decreased significantly, whereas alcohol is now the main risk factor for cirrhosis, while NAFLD has emerged as a continually increasing disorder associated with both cirrhosis and HCC. In the coming years, the epidemiology of HCC is expected to continue its transformation and metabolic risk factors could grow to be the dominant risk factor for cirrhosis and HCC. Healthcare and prevention programs for cirrhosis and HCC should take these future trends into account.

\section{References}

1. European Association for Study of Liver. EASL Clinical Practice Guidelines: management of hepatitis C virus infection. J Hepatol 2014;60:392-420.

2. Parkin DM, Bray F, Ferlay J, Pisani P. Global cancer statistics, 2002. CA Cancer J Clin 2005;55:74-108.

3. Fleming KM, Aithal GP, Solaymani-Dodaran M, Card TR, West J. Incidence and prevalence of cirrhosis in the United Kingdom, 19922001: a general population-based study. J Hepatol 2008;49:732-738.

4. Garcia-Tsao G, Friedman S, Iredale J, Pinzani M. Now there are many (stages) where before there was one: In search of a pathophysiological classification of cirrhosis. Hepatology 2010;51:1445-1449.

5. Nault JC. Pathogenesis of hepatocellular carcinoma according to aetiology. Best Pract Res Clin Gastroenterol 2014;28:937-947.

6. El-Serag HB. Hepatocellular carcinoma. $N$ Engl $J$ Med 2011;365:1118-1127.

7. Liou I, Kowdley KV. Natural history of nonalcoholic steatohepatitis. J Clin Gastroenterol 2006;40 Suppl 1:S11-S16.

8. Koulentaki M, Moscandrea J, Dimoulios P, Chatzicostas C, Kouroumalis EA. Survival of anti-mitochondrial antibody-positive and -negative primary biliary cirrhosis patients on ursodeoxycholic acid treatment. Dig Dis Sci 2004;49:1190-1195.

9. Fattovich G, Pantalena M, Zagni I, Realdi G, Schalm SW, Christensen E; European Concerted Action on Viral Hepatitis (EUROHEP). Effect of hepatitis B and C virus infections on the natural history of compensated cirrhosis: a cohort study of 297 patients. Am J Gastroenterol 2002;97:2886-2895.

10. Fattovich G, Stroffolini T, Zagni I, Donato F. Hepatocellular carcinoma in cirrhosis: incidence and risk factors. Gastroenterology 2004;127:S35-S50.

11. El-Serag HB. Epidemiology of viral hepatitis and hepatocellular carcinoma. Gastroenterology 2012;142:1264-1273.

12. Nordenstedt H, White DL, El-Serag HB. The changing pattern of epidemiology in hepatocellular carcinoma. Dig Liver Dis 2010;42
Suppl 3:S206-S214.

13. Hamed MA, AliSA. Non-viral factors contributing to hepatocellular carcinoma. World J Hepatol 2013;5:311-322.

14. Bosetti C, Turati F, La Vecchia C. Hepatocellular carcinoma epidemiology. Best Pract Res Clin Gastroenterol 2014;28:753-770.

15. Calle EE, Teras LR, Thun MJ. Obesity and mortality. N Engl J Med 2005;353:2197-2199.

16. El-Serag HB, Rudolph KL. Hepatocellular carcinoma: epidemiology and molecular carcinogenesis. Gastroenterology 2007;132:2557-2576.

17. Resuli B, Sallaku A. Hepatocellular carcinoma in Albania: incidence and risk factors. IJSR 2016;5:1178-1183.

18. Lionis C, Koulentaki M, Biziagos E, Kouroumalis E. Current prevalence of hepatitis A, B and C in a well-defined area in rural Crete, Greece. J Viral Hepat 1997;4:55-61.

19. Drositis I, Bertsias A, Lionis C, Kouroumalis E. Epidemiology and molecular analysis of hepatitis A, B and C in a semi-urban and rural area of Crete. Eur J Intern Med 2013;24:839-845.

20. Abu-Amara M, Feld JJ. Does antiviral therapy for chronic hepatitis B reduce the risk of hepatocellular carcinoma? Semin Liver Dis 2013;33:157-166.

21. Santi V, Buccione D, Di Micoli A, et al. The changing scenario of hepatocellular carcinoma over the last two decades in Italy. $J$ Hepatol 2012;56:397-405.

22. Raptis I, Koskinas J, Emmanouil T, Hadziyannis S. Changing relative roles of hepatitis $\mathrm{B}$ and $\mathrm{C}$ viruses in the aetiology of hepatocellular carcinoma in Greece. Epidemiological and clinical observations. J Viral Hepat 2003;10:450-454.

23. Kabiri M, Jazwinski AB, Roberts MS, Schaefer AJ, Chhatwal J. The changing burden of hepatitis $C$ virus infection in the United States: model-based predictions. Ann Intern Med 2014;161:170-180.

24. Yu ML, Chuang WL, Chen SC, et al. Changing prevalence of hepatitis $\mathrm{C}$ virus genotypes: molecular epidemiology and clinical implications in the hepatitis $\mathrm{C}$ virus hyperendemic areas and a tertiary referral center in Taiwan. J Med Virol 2001;65:58-65.

25. Raimondi S, Bruno S, Mondelli MU, Maisonneuve P. Hepatitis C virus genotype $1 \mathrm{~b}$ as a risk factor for hepatocellular carcinoma development: a meta-analysis. J Hepatol 2009;50:1142-1154.

26. Kanwal F, Kramer JR, Ilyas J, Duan Z, El-Serag HB. HCV genotype 3 is associated with an increased risk of cirrhosis and hepatocellular cancer in a national sample of U.S. Veterans with HCV. Hepatology 2014;60:98-105.

27. Ceni E, Mello T, Galli A. Pathogenesis of alcoholic liver disease: role of oxidative metabolism. World J Gastroenterol 2014;20:17756-17772.

28. Hjelkrem MC, Torres DM, Harrison SA. Nonalcoholic fatty liver disease. Minerva Med 2008;99:583-593.

29. Powell EE, Cooksley WG, Hanson R, Searle J, Halliday JW, Powell LW. The natural history of nonalcoholic steatohepatitis: a follow-up study of forty-two patients for up to 21 years. Hepatology 1990;11:74-80.

30. Rosmorduc O, Fartoux L. HCC and NASH: how strong is the clinical demonstration? Clin Res Hepatol Gastroenterol 2012;36:202-208.

31. Page JM, Harrison SA. NASH and HCC. Clin Liver Dis 2009;13:631-647.

32. Lidofsky SD. Nonalcoholic fatty liver disease: diagnosis and relation to metabolic syndrome and approach to treatment. Curr Diab Rep 2008;8:25-30.

33. Kanwal F, Kramer JR, Duan Z, Yu X, White D, El-Serag HB. Trends in the Burden of Nonalcoholic Fatty Liver Disease in a United States Cohort of Veterans. Clin Gastroenterol Hepatol 2016;14:301308.e1-e2.

34. Bedogni G, Miglioli L, Masutti F, Tiribelli C, Marchesini G, Bellentani S. Prevalence of and risk factors for nonalcoholic fatty liver disease: the Dionysos nutrition and liver study. Hepatology 2005;42:44-52. 